\title{
Probing the light Higgs pole resonance annihilation of dark matter in the light of XENON100 and CDMS-II observations.
}

\author{
Utpal Chattopadhyay $^{a}$, Debottam Das ${ }^{b, a}$, Dilip Kumar Ghosh ${ }^{a}$ and Manas Maity ${ }^{c}$ \\ ${ }^{a}$ Department of Theoretical Physics, Indian Association for the Cultivation of Science, \\ $2 A \& 3$ B Raja S.C. Mullick Road, Jadavpur, Kolkata 700 032, India \\ ${ }^{b}$ Laboratoire de Physique Théorique, UMR 8627, Université de Paris-Sud 1183 CNRS, \\ Bâtiment 210, 91405 Orsay Cedex, France \\ ${ }^{c}$ Department of Physics, Visva-Bharati, Santiniketan 731235, India
}

\begin{abstract}
We consider the prospect of lightest neutralino $\left(\tilde{\chi}_{1}^{0}\right)$ as a dark matter candidate in the light of recent interesting observations from the XENON100 and CDMS-II experiments in minimal supergravity framework with large $\tan \beta$ and nonvanishing $A_{0}$. Within the WMAP satisfied zone, there is a large direct detection reach of lighter $\tilde{\chi}_{1}^{0}$ in the lighter Higgs boson mediated resonance annihilation domain of the above scenario. It is seen that the heavier Higgs boson plays a dominating role in the $\tilde{\chi}_{1}^{0}-p$ cross section in the associated zone of parameter space in spite of having a larger mass. Possible LHC signatures are discussed.
\end{abstract}

\section{Introduction}

Low energy supersymmetry (SUSY) [1] has several features that make it very promising as a class of models for beyond the standard model (SM) of particle physics [2]. The minimal supergravity (mSUGRA) [3] is a well-studied model that greatly reduces the number of parameters of the minimal supersymmetric standard model (MSSM) $[1,4]$ through a few simple requirements. The universal input parameters given at the unification scale are: (i) the gaugino mass parameter $m_{1 / 2}$, (ii) the scalar mass parameter $m_{0}$, and (iii) the trilinear SUSY breaking parameter $A_{0}$. The model that uses radiative breaking of electroweak symmetry requires another parameter $\tan \beta$ which is the ratio of Higgs vacuum expectation values. Additionally the sign of the Higgsino mixing parameter $\mu$ is an input. With 
R-parity [4] assumed to be preserved, the model has an attractive dark matter [5,6] candidate, namely the lightest neutralino $\tilde{\chi}_{1}^{0}$ which is the lightest supersymmetric particle (LSP) for most of the parameter space. In a scenario where $\tilde{\chi}_{1}^{0}$ is the LSP, the parameter space with other sparticles lighter than $\tilde{\chi}_{1}^{0}$ are ignored. The model is typically associated with a Bino-dominated LSP that leads to over-abundance of dark matter. Reduction of the relic density to satisfy the WMAP data can be achieved if i) there is coannihilation of LSP with another sfermion (usually stau $\tilde{\tau}_{1}$ or rarely $\tilde{t}_{1}$ ) that has mass close to the mass of LSP [7], ii) there is appropriate mixture of Bino and Higgsinos in the composition of the LSP so that there may be coannihilating charginos in the LSP- $\tilde{\chi}_{1}^{ \pm}$annihilation $[8,9]$, the so called focus point [10]/hyperbolic branch [11] region, iii) the LSP is sufficiently small in mass and sfermions are light so that light sfermion exchange may enhance the LSP-LSP annihilation rates, or iv) there is a possibility of having s-channel Higgs exchanges where the exchanges may occur via CP-odd Higgs boson $A$ or via CP-even heavy (light) Higgs bosons $H(h)$ leading to the "funnel region" of dark matter satisfied zone in the $m_{1 / 2}-m_{0}$ plane [12].

Most recently XENON100 [13] experiment has published an updated result for the exclusion limit of $3 \times 10^{-8} \mathrm{pb}$ for direct search of dark matter for a weakly interacting massive particle (WIMP) mass of $50 \mathrm{GeV}$ at $90 \%$ confidence limit. Additionally, the recent 5-tower result of the CDMS-II [14] experiment published a result of 2 signal events with $0.6 \pm 0.1$ events as background. As mentioned by the authors, there is a significant chance that these two events might have been caused by background rather than by any real signal. However, a low recoil of 12.3 to $15.5 \mathrm{keV}$ [14] possibly suggests a lighter WIMP with a mass not quite far from $100 \mathrm{GeV}$. We may thus assume that the spin-independent direct detection cross section limit $\left(\sigma_{\tilde{\chi}_{1}^{0} p}^{S I}\right)$ set by the experiment, namely $3.8 \times 10^{-8}$ pb at a WIMP mass in the vicinity of $70 \mathrm{GeV}$ may become an interesting zone in the near future in a light LSP scenario. Several analyses have used this theme in the recent past [15] and there have been several recent works [16] in SUSY models in the context of the CDMS-II result. For practical purposes, we will probe the region of $5 \times 10^{-9}$ to $5 \times 10^{-8}$ pb in a lighter LSP scenario (not too far from $100 \mathrm{GeV}$ ) that satisfies the following relic density bounds from WMAP [17]:

$$
\Omega_{\tilde{\chi}_{1}^{0}} h^{2}=0.1099 \pm 0.0186(3 \sigma) .
$$

A possibly preferred scenario where the LSP is light and at the same time is dominated by Bino may be achieved if neutralino relic density is brought to an acceptable level (from a typical over-abundance) via a light Higgs pole annihilation mechanism. Computation of 
relic density in mSUGRA with nonvanishing trilinear coupling for large $\tan \beta$ showed such a possibility in Ref. [18] that also imposed constraints from flavor physics like that from $b \rightarrow s \gamma$ and $B_{s} \rightarrow \mu^{+} \mu^{-}$. Here in this work, we will particularly explore the reach of the direct detection cross section in relation to any small recoil data of CDMS-II in such a scenario with light Higgs pole annihilation. In addition to imposing the LEP2 limit [19] for $m_{h}$, the mass of the light Higgs boson, we will also explore the parameter space in regard to the constraints like $b \rightarrow s \gamma$ and $B_{s} \rightarrow \mu^{+} \mu^{-}$processes. We will particularly discuss the effect of considering nonminimal flavor violating (non-MFV) scenarios with a wider point of view that may be considered without affecting much the spectra or the dark matter related results. We will further probe the possible signatures coming out of LHC in regard to MSSM Higgs bosons and weakly interacting gauginos $\left(\tilde{\chi}_{2}^{0}, \tilde{\chi}_{1}^{ \pm}\right)$in the dark matter satisfied zone with larger direct detection cross section $\sigma_{\tilde{\chi}_{1}^{0} p}^{S I}$. We note that LHC may help to probe the mass of the lighter Higgs boson $\left(m_{h}\right)$ from $p p \rightarrow h \rightarrow \gamma \gamma$ mode, whereas an existence of light Higgs pole annihilation would indicate a light LSP scenario with a value of $m_{\widetilde{\chi}_{1}^{0}}$ that would satisfy $2 m_{\widetilde{\chi}_{1}^{0}} \lesssim m_{h}$. Additionally, at the LHC, one would expect to see a signal of three leptons and a missing transverse energy namely $3 \ell+E_{T}$ mainly from the pair production of $\tilde{\chi}_{2}^{0} \tilde{\chi}_{1}^{ \pm}$followed by their decays via leptonic modes, $p p \rightarrow \tilde{\chi}_{2}^{0} \tilde{\chi}_{1}^{ \pm} \rightarrow 3 \ell+E_{T}$ as a classic signature of SUSY. We also note that in regard to the decay $\tilde{\chi}_{2}^{0} \rightarrow \ell^{+} \ell^{-} \tilde{\chi}_{1}^{0}$, the endpoint of opposite sign same flavor (OSSF) dilepton invariant mass distribution $m_{\ell^{+} \ell^{-}}$leads to a good determination of the mass difference $m_{\tilde{\chi}_{2}^{0}}-m_{\tilde{\chi}_{1}^{0}}[20,21]$.

This article is organized as follows. In Sec. 2, we discuss the issues of direct detection of dark matter particularly the spin-independent cross section $\sigma_{\tilde{\chi}_{1}^{0} p}^{S I}$ in relation to the parameter space of mSUGRA that satisfies the WMAP data via s-channel Higgs pole annihilation. In Sec. 3, we identify the preferred sign of the trilinear coupling in relation to the above schannel annihilation. In Sec. 4, we discuss the implications of WMAP data and low energy constraints on the mSUGRA parameter space that is interesting for a CDMS-II type of event with a small LSP mass. In Sec. 5, we discuss several possible signatures of this scenario at the LHC. We also comment on how the correlations between different LHC signatures and the direct detection of dark matter may help determine the masses of $\tilde{\chi}_{1}^{0}$ and $\tilde{\chi}_{2}^{0}$. Finally, we summarise our findings in Sec. 6 . 


\section{Direct detection of dark matter}

In general, the results of cross section of LSP annihilation to quarks can be used to find the elastic scattering cross section of the LSP with quark, thanks to crossing symmetry. Direct detections of LSPs involve measurement of recoil energy of a nucleus due to LSP-nucleon scattering $[6,22,23]$. Typical detector materials are scintillators like NaI, semiconductors like Ge and noble liquids like Xe. The elastic scattering of neutralino with nucleons are divided into two types (i) spin-independent (SI): a neutralino coherently interacts with the nucleus and (ii) spin-dependent (SD): a neutralino interacts with matter via axial vector coupling. The effective Lagrangian that describes $\chi-q$ elastic scattering for a small velocity is given by,

$$
\mathcal{L}=\alpha_{q i}^{\prime} \bar{\chi} \gamma^{\mu} \gamma^{5} \chi \bar{q}_{i} \gamma_{\mu} \gamma^{5} q_{i}+\alpha_{q i} \bar{\chi} \chi \bar{q}_{i} q_{i}
$$

Here, the first term represents spin-dependent scattering while the second term refers to spin-independent scattering. The terms assume summing over the quark flavors $q$ as well as that for up and down type of quarks (for $i=1$ and $i=2$ respectively). The neutralino-quark coupling coefficients $\alpha_{q}$ and $\alpha_{q}^{\prime}$ contain all SUSY model dependent information [22]. The spin-independent scattering cross section of a neutralino with a target nucleus of proton number (atomic number) $Z$ and neutron number $A-Z$ ( $A$ being the mass number) is given by,

$$
\sigma^{S I}=\frac{4 m_{r}^{2}}{\pi}\left[Z f_{p}+(A-Z) f_{n}\right]^{2} .
$$

Here, $m_{r}$ is the reduced mass defined by $m_{r}=\frac{m_{\chi} m_{N}}{\left(m_{\chi}+m_{N}\right)}$, where $m_{N}$ refers to the mass of the nucleus. The quantities $f_{p}$ and $f_{n}$ contain all the information of short-distance physics and nuclear partonic strengths. These are given by,

$$
\frac{f_{p,(n)}}{m_{p,(n)}}=\sum_{q=u, d, s} f_{T q}^{(p,(n))} \frac{\alpha_{q}}{m_{q}}+\frac{2}{27} f_{T G}^{(p,(n))} \sum_{c, b, t} \frac{\alpha_{q}}{m_{q}} .
$$

$f_{T q}^{(p,(n))}$ and $f_{T G}^{(p,(n))}$ refer to interactions of neutralino with quarks and gluons (via quark/squark loop diagrams) respectively. For $f_{T q}^{(p,(n))}$ one has,

$$
m_{p,(n)} f_{T q}^{(p,(n))}=\left\langle p,(n)\left|m_{q} \bar{q} q\right| p,(n)\right\rangle \equiv m_{q} B_{q} .
$$

The quantities $B_{q}$ may be computed from a few hadronic mass data. The gluon related part namely $f_{T G}^{(p,(n))}$ is given by [22,23],

$$
f_{T G}^{(p,(n))}=1-\sum_{q=u, d, s} f_{T q}^{(p,(n))} .
$$


The numerical values of $f_{T q}^{(p,(n))}$ may be seen in Ref. [22]. We compute $\sigma^{S I}$ by using the code DarkSusy [24]. In contrast to $\sigma^{S I}$, the spin-dependent cross section denoted by $\sigma^{S D}$ (for scattering of LSP with the target nucleus) does not depend on $A$ or $Z$, rather it scales with $J(J+1)$ where $J$ is the total nuclear spin. In general, the spin-independent neutralinonucleon scattering cross sections (where $\sigma_{\chi p}^{S I} \simeq \sigma_{\chi n}^{S I}$ ) are appreciably smaller than the corresponding spin-dependent cross sections $\left(\sigma_{\chi p}^{S D} \simeq \sigma_{\chi n}^{S D}\right)$. However considering the fact that $\sigma^{S D} \propto J(J+1)$ and $\sigma^{S I} \propto Z^{2},(A-Z)^{2}$ one finds that $\sigma^{S I}$ to be considerably larger for moderately heavy elements $(A>30)$ like Xe, Ge etc. The experiments like EDELWEISS [25] and CDMS [15] use natural germanium (almost purely spinless) as the target material and these hardly have any spin-sensitivities [26]. It is important to know the composition of the $\widetilde{\chi}_{1}^{0}$ in terms of Bino, Wino and Higgsinos in MSSM while analyzing the WMAP satisfied relic density region as well as the LSP-nucleon scattering cross section for a direct detection. The amount of Higgsino mixing in a typically Bino-dominated LSP scenario of mSUGRA depends on the relative value of $|\mu|$ and $m_{1}$ (the mass of Bino). The scalar cross section depends on t-channel Higgs exchange $(h, H)$ and s-channel squark exchange diagrams. Unless, the squark masses are close to the mass of the LSP, the Higgs exchange diagrams usually dominate over the s-channel diagrams [27]. We focus on a decoupling region of Higgs [28], so that there is a considerable difference of masses between $h$ and $H$-bosons. We also focus on the case where LSP is principally composed of Bino, $\tan \beta$ is large and there is a resonance annihilation via $h$-boson that is consistent with the relic density from the WMAP limits. Following the results of Refs. $[27,29]$ we see that both the contributions involving lighter as well as heavier Higgs bosons in the direct detection are important. Indeed in the characteristic zone of the parameter space as mentioned above, the heavier Higgs boson contribution becomes dominant in comparison to the contribution from the lighter Higgs boson [30]. This holds in spite of the fact that $m_{H}$ can be larger by a factor of 3 to 4 compared to $m_{h}$. In this light Higgs annihilation zone we will see that $\sigma_{\tilde{\chi}_{1}^{0} p}^{S I}$ can be large enough to reach the CDMS-II limit. 


\section{Preference of negative $A_{0}$ for light Higgs-pole reso- nance}

In this analysis we especially investigate the parameter space that satisfies the relic density limits from WMAP data via LSP annihilations through light Higgs resonance. This necessitates a closer study of the region with small $m_{1 / 2}$ near its domain just allowed by the LEP2 limit of lighter chargino mass $m_{\tilde{\chi}_{1}^{ \pm}}[31]$. We will see how the LEP2 constraint affects the lowest possible value of $m_{1 / 2}$ in a nonvanishing $A_{0}$ scenario $^{1}$. This in turn requires a discussion on the behaviors of i) $\mu$ and ii) $m_{2}{ }^{2}$ for a variation of $A_{0}$. We note that in mSUGRA the variation of $\mu$ with $A_{0}$ has the following pattern. For $A_{0}<0,|\mu|$ increases with $\left|A_{0}\right|$, whereas $|\mu|$ increases with $A_{0}$ as long as $A_{0} \geq m_{1 / 2}$. On the other hand, $\mu$ is near its minimum when one has $A_{0} \lesssim m_{1 / 2}$. As a result, one finds that for a given set of $m_{0}$, $m_{1 / 2}$ and $\tan \beta$ a change of $A_{0}$ from $A_{0}=x$ to $A_{0}=-x$ where $x \sim \mathcal{O}$ TeV causes $|\mu|$ to vary from a large to a larger value. This in turn causes $\tilde{\chi}_{1}^{ \pm}$to be dominated by Wino in the region with a smaller $m_{1 / 2}$, the amount of Wino content is larger for the case of $A_{0}=-x$ in comparison to that of $A_{0}=x$ where the same content is still significantly large. Thus, while probing the region of small gaugino masses with Wino-dominated $\tilde{\chi}_{1}^{ \pm}$, it is important to examine the variation of $m_{2}$ with nonvanishing $A_{0}$. In this context, Table 1 shows the masses of electroweak gauginos, the value of higgsino mass parameter, the masses of lighter chargino, lighter neutralino and h-boson for $\tan \beta=50, m_{1 / 2}=150 \mathrm{GeV}, m_{0}=750 \mathrm{GeV}$ and $\mu>0$ for $A_{0}= \pm 1250 \mathrm{GeV}$. This will indeed demonstrate the effect of two-loop RGEs along with threshold corrections on the electroweak gaugino masses. Clearly, both $m_{1}$ and $m_{2}$ are smaller for $A_{0}=x$ in comparison to the same quantities corresponding to $A_{0}=-x$. On the other hand, the values of $\mu$ for both signs of $A_{0}$ are sufficiently large so that the lighter chargino as well as the lighter neutralino are both highly gaugino-dominated. The value of $m_{h}$ is smaller for $A_{0}>0$. Thus, as we decrease $m_{1 / 2}$, the LEP2 bound of chargino mass is reached at a relatively larger $m_{1 / 2}$ for $A_{0}=x$ in comparison to the case of $A_{0}=-x$. Similarly, the LEP2 limit of $m_{h}$ is reached at a relatively larger $m_{1 / 2}$ for $A_{0}=x$ than the case of $A_{0}=-x$. The latter favors larger loop corrections from the top-stop sector [33].

\footnotetext{
${ }^{1}$ We have imposed restrictions to avoid the appearance of charge and color breaking (CCB) minima [32] in the parameter space.

${ }^{2}$ We consider two-loop RGEs along with threshold corrections.
} 
In brief, imposition of LEP2 bounds of $m_{\tilde{\chi}_{1}^{ \pm}}$and $m_{h}$ (considering also the aforesaid $3 \mathrm{GeV}$ theoretical uncertainty) would allow us to reach to a smaller $m_{1 / 2}$ value for a negative $A_{0}$ than for a positive $A_{0}$ [34]. As a result, Bino can be light enough to satisfy a light Higgspole annihilation condition $2 m_{\widetilde{\chi}_{1}^{0}} \lesssim m_{h}$ while obeying the WMAP limits in a LEP2 satisfied parameter zone. Such a light LSP would undoubtedly be interesting in view of the CDMS-II results with two possible events.

\begin{tabular}{|c|c|c|c|c|c|c|c|c|c|c|c|}
\hline \multicolumn{5}{|c|}{$A_{0}=1250.0 \mathrm{GeV}$} & \multicolumn{5}{c|}{$A_{0}=-1250.0 \mathrm{GeV}$} \\
\hline$m_{1}$ & $m_{2}$ & $\mu$ & $m_{\tilde{\chi}_{1}^{ \pm}}$ & $m_{\tilde{\chi}_{1}^{0}}$ & $m_{h}$ & $m_{1}$ & $m_{2}$ & $\mu$ & $m_{\tilde{\chi}_{1}^{ \pm}}$ & $m_{\tilde{\chi}_{1}^{0}}$ & $m_{h}$ \\
\hline 58.8 & 111.2 & 352.4 & 104.7 & 57.5 & 106.6 & 62.4 & 118.7 & 492.3 & 121.9 & 62.2 & 114.3 \\
\hline
\end{tabular}

Table 1: Comparison of spectra electroweak gaugino masses, higgsino masses, the masses of lighter chargino, lighter neutralino and h-boson for $\tan \beta=50, m_{1 / 2}=150 \mathrm{GeV}, m_{0}=$ $750 \mathrm{GeV}$ and $\mu>0$ for $A_{0}= \pm 1250 \mathrm{GeV}$. This is given as an example to demonstrate the effect of two-loop RGEs along with threshold corrections on the electroweak gaugino masses which are smaller for $A_{0}>0$. These are of course not valid parameter points satisfying WMAP data for neutralino relic density.

\section{WMAP data and low energy constraints}

We now explain the results of imposing dark matter constraint for $A_{0}<0$ for large $\tan \beta$ and the relevance of the light-Higgs pole mediated annihilations in relation to the CDMS-II results. Fig. 1 shows the results for $\mu>0$ in $m_{0}-m_{1 / 2}$ plane for $\tan \beta=50$ and $A_{0}=$ $-1250 \mathrm{GeV}^{3}$. Almost the entire parameter space has a highly Bino-dominated LSP except for the region where $m_{0}$ is large. The latter region is associated with a significant amount of higgsinos in the composition of the LSP. The WMAP constraint of Eq.1 is satisfied in the blue shaded regions which are divided into three broad zones. The lower blue region with $m_{1 / 2}<900 \mathrm{GeV}$ and $m_{0}<1 \mathrm{TeV}$ satisfies WMAP data via LSP-stau coannihilation. The extension of this blue region in the larger $m_{1 / 2}$ side is associated with LSP-LSP resonance annihilation via $A / H$-bosons. The upper blue $m_{0}$ region satisfying the WMAP limits is

\footnotetext{
${ }^{3}$ We consider top pole mass $m_{t}=171.4 \mathrm{GeV}$.
} 


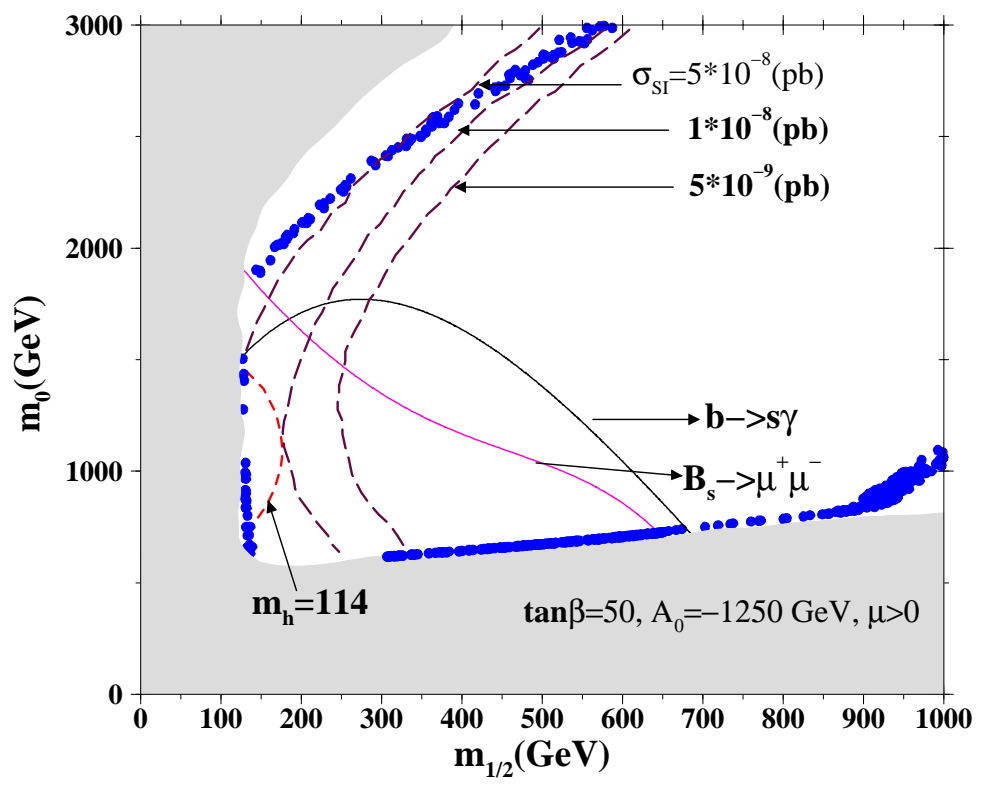

Figure 1: Results for $\tan \beta=50$ and $A_{0}=-1250 \mathrm{GeV}$ for $\mu>0$. The WMAP satisfied zones in $m_{0}-m_{1 / 2}$ plane are shown in blue. The lower blue region with $m_{1 / 2}<900 \mathrm{GeV}$ and $m_{0}<1 \mathrm{TeV}$ satisfies WMAP data via LSP-stau coannihilation. The extension of this blue region in the larger $m_{1 / 2}$ side is associated with LSP-LSP resonance annihilation via $A / H$-bosons. The left-most vertical blue region with small $m_{1 / 2}<150 \mathrm{GeV}$ corresponds to $s$-channel annihilation via light Higgs boson $h$. Contours shown for $\sigma_{S I}^{\tilde{\chi} p}$ from $5 \times 10^{-9} \mathrm{pb}$ to $5 \times 10^{-8} \mathrm{pb}$. The left hand sides of the contours for $\operatorname{Br}(b \rightarrow s \gamma)$ and $B_{s} \rightarrow \mu^{+} \mu^{-}$are discarded in the minimal flavor violation scenario. We have considered the possibility of a general flavor violation scenario as mentioned in the text. The details of the discarded regions are mentioned in the text. 
characterised by a considerably large Higgsino fraction in the composition of LSP. Here the $s$-channel annihilation occurs via $A / H$-bosons that may be several widths $\left(\Gamma_{A, H}\right)$ away. The left-most blue region with small $m_{1 / 2}$ corresponds to $s$-channel annihilation via light Higgs boson $h$. Bracketing the sensitivity level of the CDMS-II result in particular $3.8 \times 10^{-8} \mathrm{pb}$ or the XENON100 data of $3 \times 10^{-8}$ pb we have drawn contours of the spin-independent $\tilde{\chi} p$ cross section $\sigma_{S I}^{\tilde{\chi} p}$ from $5 \times 10^{-9} \mathrm{pb}$ to $5 \times 10^{-8} \mathrm{pb}$. We see that apart from the large $m_{0}$ region satisfying the WMAP data via $A / H$ resonance, there exists a significant possibility to probe the region of light Higgs boson resonance that produces a large $\sigma_{S I}^{\tilde{\chi} p}$. We also show a contour of $m_{h}=114 \mathrm{GeV}$ which is close to the LEP2 bound of $h$ of $114.4 \mathrm{GeV}$. We note that although we have considered a large value for $\tan \beta$ the pseudoscalar Higgs boson is not very light. Thus the latter being still in the decoupling region the associated lighter Higgs boson $h$ is standard model like. However, we should also note that there is about a $3 \mathrm{GeV}$ uncertainty in the theoretical result of $m_{h}$ while including the loop corrections [35]. Hence we consider a value of $m_{h}=111 \mathrm{GeV}$ to be an effective lower limit. We further draw contours corresponding to the limits from $\operatorname{Br}(b \rightarrow s \gamma)[36-38]$ and $\operatorname{Br}\left(B_{s} \rightarrow \mu^{+} \mu^{-}\right)[39,40]$. The limits for $\operatorname{Br}(b \rightarrow s \gamma)$ at $3 \sigma$ level is given by [38]

$$
2.77 \times 10^{-4}<\operatorname{Br}(b \rightarrow s \gamma)<4.33 \times 10^{-4} .
$$

Clearly, the above limits of $b \rightarrow s \gamma$ are not favorable to the light Higgs pole annihilation region satisfying the WMAP constraint. We however point out that the above limits apply to models where perfect alignment of the squark and quark mass matrices are assumed. This indicates no extra mixing other than the Cabibbo-Kobayashi-Maskawa factors existing at the corresponding SM vertices. However, one may obtain a considerable degree of squark mixing at the electroweak scale if there is even a very small off-diagonal term at the grand unification scale. As discussed in Ref. [41] this may considerably reduce the effect of the constraint from $b \rightarrow s \gamma$ on the parameter space of the SUSY model. On the other hand, such a possible deviation from the minimal flavor violation (MFV) [42] scenario does not cause any significant change in the sparticle masses or in the results of flavor conserving processes like neutralino annihilation. Additionally, limits from $\operatorname{Br}\left(B_{s} \rightarrow \mu^{+} \mu^{-}\right)$becomes important in a large $\tan \beta$ and/or small pseudoscalar Higgs mass scenario since $\operatorname{Br}\left(B_{s} \rightarrow \mu^{+} \mu^{-}\right)$varies as $\tan ^{6} \beta$ or in a light pseudoscalar Higgs boson scenario the same varies as $m_{A}^{-4}$ [39]. The present upper limit is given below [40].

$$
\operatorname{Br}\left(B_{s} \rightarrow \mu^{+} \mu^{-}\right)<5.8 \times 10^{-8} .
$$


We may as well consider a non-MFV scenario of squark mixing for $B_{s} \rightarrow \mu^{+} \mu^{-}$and thereby consider the light Higgs pole annihilation region to be an open region for study. References. [43-45] may be seen for analyses with non-MFV scenarios for $\operatorname{Br}(b \rightarrow s \gamma)$ and $B_{s} \rightarrow \mu^{+} \mu^{-}$. Following the arguments and analyses of Refs. [41,43-45] we therefore prefer not to exclude any region allowed by the WMAP data on the basis of the above constraints from flavor physics. The discarded regions are shown in gray. The bottom gray region is discarded via staus $\left(\tilde{\tau}_{1}\right)$ becoming the LSP. The left gray region with smaller $m_{0}$ with almost a vertical boundary is discarded via the LEP2 chargino mass bound requirement $m_{\tilde{\chi}^{ \pm}}>103.5 \mathrm{GeV}$. The top left gray region is discarded because of absence of radiative electroweak symmetry breaking.

Finally for completeness we show Fig.2(a) and Fig.2(b) in regard to the results corresponding to a vanishing and a positive $(=1250 \mathrm{GeV}) A_{0}$ respectively. Unlike Fig.1 these two cases do not have any light Higgs pole annihilation region. The s-channel annihilations occur only via $A / H$ bosons for these two cases.

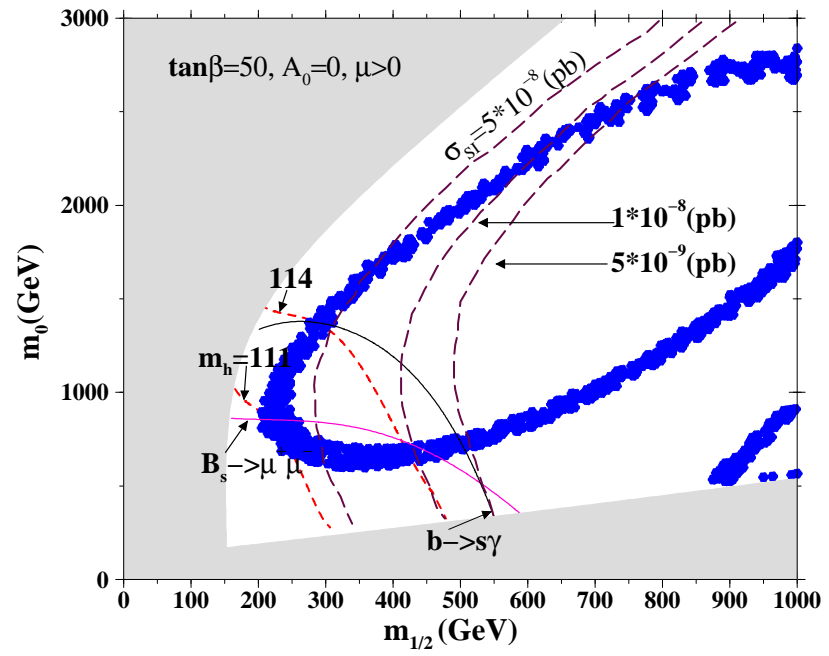

(a)

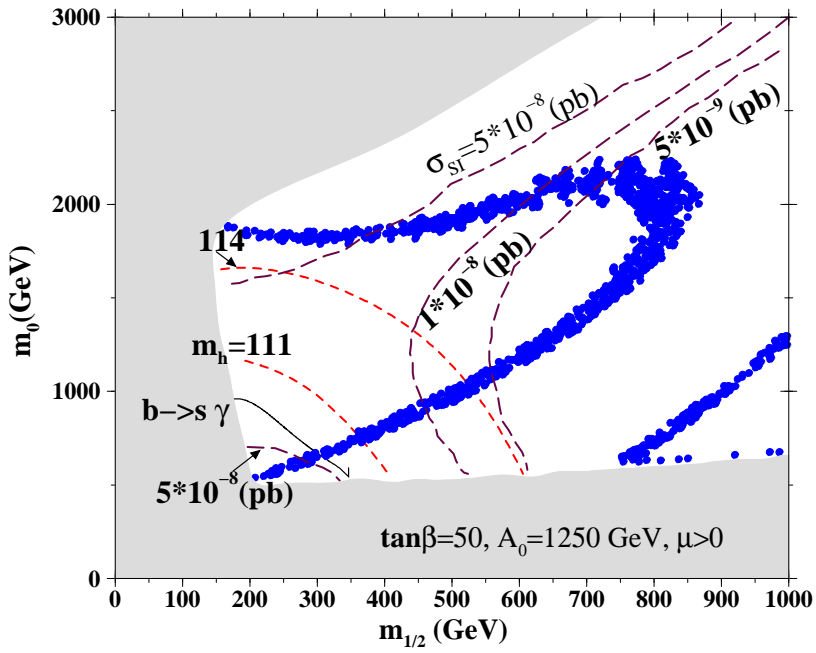

(b)

Figure 2: Similar to Fig.1 except for $A_{0}=0$ and $A_{0}=1250 \mathrm{GeV}$ as shown above. There are no light Higgs resonance regions in these two cases. 


\section{$5 \quad$ LHC phenomenology}

We now qualitatively discuss a few issues regarding various LHC searches for the Higgs bosons as well as strongly and weakly interacting gauginos in our scenario where LSP-LSP annihilation mechanism related to dark matter relics occurs via the light Higgs resonance channel. We present two benchmark points namely A and B in Table 2 with the corresponding SUSY spectra in this regard. Detailed analyses for the searches will be reported in Ref. [46]. The benchmark points satisfy the necessary phenomenological constraints including the LEP2 bound for the Higgs boson mass except the fact that they do not satisfy the limits from $\operatorname{Br}(b \rightarrow s \gamma)$ and $B_{s} \rightarrow \mu^{+} \mu^{-}$, if minimal flavor violation is assumed. As mentioned before, the above flavor constraints are relaxed in a general or a non-MFV type of estimation. The leading decay modes along with the branching ratios (BR) corresponding to the points $\mathrm{A}$ and $\mathrm{B}$ are given in Table 3. We note that the above points obey the decoupling limit $\left(m_{A}>>M_{Z}\right)$ so that the lighter Higgs boson $h$ behaves like a standard model like Higgs boson. We see that WMAP satisfied zone corresponding to the light Higgs pole annihilation is very close to the exclusion limit provided by the LEP2 and Tevatron. Being in a decoupling zone, the pseudoscalar Higgs boson $(A)$ is appreciably massive with also having a large width $\left(\Gamma_{A}\right)$. Thus it can hardly have a simultaneous contribution to the resonance annihilation in a parameter zone where there is a light Higgs boson in the s-channel. On the other hand with a small width $\Gamma_{h}$ for the h-boson, the LSP mass $m_{\chi_{1}^{0}}$ has a tight constraint, namely $2 m_{\chi_{1}^{0}} \simeq m_{h}$ from the WMAP data. This particular feature when coupled with different observables related to the accelerator physics may provide a very useful estimate for the supersymmetric mass spectrum directly from the experimental data.

Before going into details of the LHC signatures predicted by this scenario, let us first discuss few salient features of the low energy SUSY spectrum which can be inferred from the Table 2.

- Large $\tan \beta$.

- The Higgs sector falls within the decoupling regime, with moderately heavy $H$ and $A$, and light SM like Higgs boson $h$.

- Light LSP mass, $m_{\widetilde{\chi}_{1}^{0}} \approx m_{h} / 2$, while lighter chargino $\left(\tilde{\chi}_{1}^{ \pm}\right)$and second lightest neutralino $\left(\tilde{\chi}_{2}^{0}\right)$ are almost degenerate with masses $\sim 105 \mathrm{GeV}$. 


\begin{tabular}{|l|c|c|}
\hline parameters & $\mathrm{A}$ & $\mathrm{B}$ \\
\hline $\tan \beta$ & 50.0 & 50.0 \\
$m_{1 / 2}$ & 132.0 & 130.0 \\
$m_{0}$ & 750.0 & 915.0 \\
$A_{0}$ & -1250.0 & -1250. \\
$s g n(\mu)$ & 1 & 1 \\
\hline \hline$\mu$ & 476.63 & 461.94 \\
$m_{\tilde{g}}$ & 384.15 & 389.54 \\
$m_{\tilde{u}_{L}}$ & 793.86 & 944.17 \\
$m_{\tilde{t}_{1}}$ & 286.49 & 425.17 \\
$m_{\tilde{t}_{2}}$ & 550.38 & 638.94 \\
$m_{\tilde{b}_{1}}$ & 453.97 & 570.91 \\
$m_{\tilde{b}_{2}}$ & 592.56 & 701.47 \\
$m_{\tilde{e}_{L}}$ & 753.03 & 915.73 \\
$m_{\tilde{\tau}_{1}}$ & 373.21 & 539.54 \\
$m_{\widetilde{\chi}_{1}^{ \pm}}$ & 107.51 & 106.72 \\
$m_{\widetilde{\chi}_{2}^{ \pm}}$ & 486.15 & 474.13 \\
$m_{\widetilde{\chi}_{4}^{0}}$ & 483.47 & 471.43 \\
$m_{\widetilde{\chi}_{3}^{0}}$ & 479.13 & 466.90 \\
$m_{\widetilde{\chi}_{2}^{0}}$ & 107.54 & 106.75 \\
$m_{\widetilde{\chi}_{1}^{0}}$ & 54.67 & 54.15 \\
$m_{A}$ & 336.09 & 352.60 \\
$m_{H^{+}}$ & 346.84 & 363.02 \\
$m_{h}$ & 113.87 & 113.0 \\
$\Omega_{\widetilde{\chi}_{1}^{0}} h^{2}$ & 0.120 & 0.114 \\
$\sigma_{S I}(p b)$ & $2.75 \times 10^{-8}$ & $2.26 \times 10^{-8}$ \\
\hline
\end{tabular}

Table 2: Benchmark points $\mathrm{A}$ and $\mathrm{B}$ along with the spectra. Masses are shown in GeV.

- Heavy first two generations of squarks and sleptons with masses above $700 \mathrm{GeV}$.

- Light gluinos $\left(m_{\tilde{g}} \leq 400 \mathrm{GeV}\right)$ and lighter third generation of squarks and sleptons. 


\begin{tabular}{|c|c|c|}
\hline $\begin{array}{c}\text { Decay modes } \\
\text { (squark/gluino) }\end{array}$ & $\mathrm{A}$ & $\mathrm{B}$ \\
\hline \hline$\widetilde{g} \rightarrow \widetilde{\chi}_{2}^{0} b \bar{b}$ & 44.0 & 36.0 \\
\hline$\widetilde{g} \rightarrow \widetilde{\chi}_{1}^{ \pm} t b$ & 14.0 & 12.0 \\
\hline$\widetilde{g} \rightarrow \widetilde{\chi}_{1}^{ \pm} u d$ & 18.0 & 24.0 \\
\hline \hline$\widetilde{b}_{1} \rightarrow \widetilde{g}^{\prime} b$ & 38.0 & 66.0 \\
\hline$\widetilde{b}_{1} \rightarrow \widetilde{\chi}_{2}^{0} b$ & 17.0 & 10.0 \\
\hline$\widetilde{b}_{1} \rightarrow \widetilde{\chi}_{1}^{ \pm} t$ & 23.0 & 15.40 \\
\hline$\widetilde{b}_{1} \rightarrow \widetilde{t}_{1} W^{-}$ & 17.0 & 5.30 \\
\hline \hline$\widetilde{t}_{1} \rightarrow \widetilde{\chi}_{1}^{+} b$ & 72.40 & 53.70 \\
\hline$\widetilde{t}_{1} \rightarrow \widetilde{\chi}_{1}^{0} t$ & 22.0 & 29.40 \\
\hline$\widetilde{t}_{1} \rightarrow \widetilde{\chi}_{2}^{0} t$ & 5.0 & 16.80 \\
\hline \hline$\widetilde{\chi}_{2}^{0} \rightarrow \widetilde{\chi}_{1}^{0} q \bar{q}$ & 48.0 & 50.0 \\
\hline$\widetilde{\chi}_{2}^{0} \rightarrow \widetilde{\chi}_{1}^{0} b \bar{b}$ & 33.0 & 28.60 \\
\hline$\widetilde{\chi}_{2}^{0} \rightarrow \widetilde{\chi}_{1}^{0} \ell^{+} \ell^{-},(\ell=e, \mu)$ & 2.54 & 3.7 \\
\hline$\widetilde{\chi}_{2}^{0} \rightarrow \widetilde{\chi}_{1}^{0} \tau^{+} \tau^{-}$ & 8.7 & 4.56 \\
\hline$\widetilde{\chi}_{2}^{0} \rightarrow \widetilde{\chi}_{1}^{0} \nu \bar{\nu}$ & 6.0 & 10.0 \\
\hline$\widetilde{\chi}_{1}^{+} \rightarrow \widetilde{\chi}_{1}^{0} u \bar{d}$ & 69.0 & 68.60 \\
\hline$\widetilde{\chi}_{1}^{+} \rightarrow \widetilde{\chi}_{1}^{0} \ell^{+} \nu_{\ell}$ & 20 & 20.0 \\
\hline$\widetilde{\chi}_{1}^{+} \rightarrow \widetilde{\chi}_{1}^{0} \tau^{+} \nu_{\tau}$ & 11.0 & 10.0 \\
\hline
\end{tabular}

Table 3: The branching ratios(\%)of the dominant decay modes of the gluinos, squarks and lighter electroweak gauginos in mSUGRA for the points A and B.

We start our discussions with the light MSSM Higgs boson $h$, which as mentioned before behaves like a SM Higgs boson. The dominant production mechanism of $h$ is via $g g \rightarrow h$ process followed by (i) $q q h$ production through vector boson fusion, (ii) associated productions with $W^{ \pm}$or $Z$ bosons, and (iii) in association with a $t \bar{t}$ pair. The $h \rightarrow \gamma \gamma$ decay mode is the best channel to look for a light Higgs boson $h$ channel with $m_{h}<130 \mathrm{GeV}[20,47]$. For the Higgs mass of our interest $(\sim 115 \mathrm{GeV})$, one has the $\sigma(p p \rightarrow h)_{\text {inclusive }} \times \mathrm{BR} \sim \mathcal{O}(100)$ $\mathrm{fb}$ and for a $5 \sigma$ discovery one requires integrated luminosity of $30 \mathrm{fb}^{-1}$ at the $14 \mathrm{TeV}$ LHC 
$\operatorname{run}[47]$.

We now turn our focus on the production of heavy neutral MSSM Higgs bosons. The bottom quark Yukawa couplings to both $H$ and $A$ become strongly enhanced at large $\tan \beta$. At the LHC, the production process of $\Phi,(\Phi \equiv H, A)$ in association with bottom quark benefits from a huge enhancement factor of $\tan ^{2} \beta$ compared to the SM case. Motivated by this large enhancement of the cross section, several analyses have been performed to study the production process of $\Phi$ either from bottom quark fusion $b \bar{b} \rightarrow \Phi$ [48-52], or in association with one or two $b$-quarks with high transverse momentum $\left(p_{T}\right)$. These authors [53-57] studied $b g \rightarrow b \Phi$ process both in the context of Tevatron and the LHC. On the other hand, $\Phi$ can be produced in association with two high $p_{T} b$-quarks through the leading order sub process $g g \rightarrow b \bar{b} \Phi[48,58-61]$. However, the above process turned out to be less promising than Higgs production with a single high $p_{T}$ b-quark at the LHC [55]. It has been shown that the discovery of heavy Higgs bosons through $b g \rightarrow b \Phi \rightarrow b \tau^{+} \tau^{-}$mode at large $\tan \beta$ is very promising at the LHC [62-64]. The Higgs mass can be reconstructed in the $\Phi \rightarrow \tau^{+} \tau^{-}$ channel from the momenta of the visible decay products of tau, i.e., either leptons or jets and the missing transverse energy $\left(E_{T}\right)$ (arising from escaping neutrinos in the $\tau$ decay), assuming that neutrinos are highly collinear due to the large boost of the $\tau$ 's [47]. Very recently another possibility of discovering $\Phi$ at the LHC with $b$-quarks has been proposed: $b g \rightarrow b \Phi \rightarrow b(b \bar{b})$ leading to $3 b$ final state [65]. Following the above discussions it is worth noting that the LHC will be able to probe the full range of $H / A$ masses given by the part of the mSUGRA parameter space that is consistent with the WMAP data satisfied via light Higgs resonance annihilation and associated with a large direct detection cross section compatible with the possible CDMS-II events.

At the LHC, multilepton + missing transverse energy final states with very little hadronic activity is one of the most promising discovery channels of supersymmetry. Such final states may come from the leptonic decay of the pair of heavy gauginos (such as $\tilde{\chi}_{1}^{ \pm}, \tilde{\chi}_{2}^{0}$ ) through real or virtual $W^{ \pm}, Z^{0}$ or via decays of sleptons to leptons and a pair of LSPs (main contributor to missing transverse energy). The electroweak production of heavy gaugino pairs

$$
\begin{aligned}
& p p \rightarrow \tilde{\chi}_{2}^{0} \tilde{\chi}_{1}^{ \pm}, \\
& \tilde{\chi}_{2}^{0} \rightarrow \tilde{\chi}_{1}^{0} \ell^{+} \ell^{-} ; \tilde{\chi}_{1}^{ \pm} \rightarrow \tilde{\chi}_{1}^{0} \ell^{ \pm} \nu_{\ell}, \quad \ell=e \text { or } \mu
\end{aligned}
$$

can lead to $3 \ell+E_{T}$ signal. The $3 \ell+E_{T}$ channel may also get some contribution from the production of heavier gauginos $p p \rightarrow \tilde{\chi}_{1}^{ \pm} \tilde{\chi}_{3}^{0}, \tilde{\chi}_{1}^{ \pm} \tilde{\chi}_{4}^{0}, \tilde{\chi}_{2}^{ \pm} \tilde{\chi}_{3}^{0}, \tilde{\chi}_{2}^{ \pm} \tilde{\chi}_{4}^{0}$ followed by their decays into 


\begin{tabular}{|c|c|c|c|}
\hline \multirow{2}{*}{$\begin{array}{c}\text { Benchmark } \\
\text { points }\end{array}$} & \multicolumn{3}{|c|}{$\sqrt{s}(\mathrm{TeV})$} \\
\cline { 2 - 4 } & 7 & 12 & 14 \\
\hline$A$ & 21.3 & 44.9 & 54.9 \\
\hline$B$ & 33.8 & 71.3 & 87.1 \\
\hline
\end{tabular}

Table 4: Leading order cross sections (fb) for $p p \rightarrow \tilde{\chi}_{2}^{0} \tilde{\chi}_{1}^{ \pm} \rightarrow 3 \ell+E_{T}$ for two benchmark points.

leptonic final states. It is worth mentioning here, that, for our choice of benchmark points $(A, B)$, the trilepton channels do not get any substantial contribution from these heavier gauginos due to smaller production cross sections and also suppressed branching ratios into leptonic final states. Hence, in our analysis, we only consider $3 \ell^{ \pm}+E_{T}$ final state arising from Eq.(9). In Table.3, we show the branching ratios of $\tilde{\chi}_{2}^{0} \rightarrow \tilde{\chi}_{1}^{0} \ell^{+} \ell^{-}$and $\tilde{\chi}_{1}^{+} \rightarrow \tilde{\chi}_{1}^{0} \ell^{+} \nu_{\ell}$ decays $(\ell=e, \mu)$. In Table 4 , we present the $\sigma\left(p p \rightarrow \tilde{\chi}_{2}^{0} \tilde{\chi}_{1}^{ \pm} \rightarrow 3 \ell+\not_{T}\right)$ without any cuts for thw two benchmark points $(A, B)$ assuming three possible values of $\sqrt{s}=7,12$ and $14 \mathrm{TeV}$ at the LHC.

In this trilepton signal i.e., $3 \ell+E_{T}$ channel, any choice of the three charged leptons $(l=e$ or $\mu$ ) must consist of an OSSF pair and an additional lepton. The end point of the OSSF dilepton invariant mass $\left(m_{\ell^{+} \ell^{-}}\right)$distribution leads to a good determination of the mass difference $\Delta m \equiv m_{\tilde{\chi}_{2}^{0}}-m_{\tilde{\chi}_{1}^{0}}[20,21]$.

On the other hand, after reconstruction of the light Higgs boson mass $m_{h}$ from $h$-decays and assuming $h$-pole annihilation as the mechanism for satisfying the WMAP data, it is possible to estimate the mass $m_{\tilde{\chi}_{1}^{0}}$, which is $m_{\tilde{\chi}_{1}^{0}} \sim m_{h} / 2$. Then the mass of $\tilde{\chi}_{2}^{0}$ may be estimated by combining $m_{\tilde{\chi}_{1}^{0}}$ and with the edge in the $m_{\ell^{+} \ell^{-}}$distribution. It may be recalled that for the present choice of benchmark points, $\tilde{\chi}_{1}^{0}$ is completely Bino-dominated, while $\tilde{\chi}_{2}^{0}$ is Wino-dominated. This leads to one to one correspondence between $U(1)$ soft gaugino mass parameter $m_{1}$ with $m_{\tilde{\chi}_{1}^{0}}$ and $S U(2)$ soft gaugino mass parameter $m_{2}$ with $m_{\tilde{\chi}_{2}^{0}}$. Hence, we expect that the knowledge of both $m_{\tilde{\chi}_{1}^{0}}$ and $m_{\tilde{\chi}_{2}^{0}}$ may be used to determine the mass parameters $m_{1}$ and $m_{2}$. It would be interesting to study whether the mass pattern of $m_{1}$ and $m_{2}$ could hint towards the gaugino mass unification which model like mSUGRA assumes.

Finally, we conclude this section, by highlighting following important observations:

1. The light Higgs boson mass can be determined with a reasonably good precision via 
$h \rightarrow \gamma \gamma$ decay mode.

2. In the present scenario, where $\tan \beta$ is large, both neutral Higgs boson masses can be determined via $H / A \rightarrow \tau \tau$ decay.

3. Both $m_{\tilde{\chi}_{1}^{0}}$ and $m_{\tilde{\chi}_{2}^{0}}$ or equivalently $m_{1}$ and $m_{2}$ can be estimated by combining the data from the direct detection of dark matter and the LHC.

4. As mentioned before, our choice of parameter space prefers light gluinos and also lighter third generation sleptons and squarks. This naturally leads to large production rate for gluinos as well as lighter stop and sbottom squarks which in turn give rise to large number of multi $b$-jet final states in association with large missing energy both at 7 and $14 \mathrm{TeV}$ LHC run. This will be another striking signature of this scenario.

\section{Conclusions}

Recent experiments like XENON and CDMS-II provide us with exclusion limits within 3-4 $\times 10^{-8} p b$ for WIMP mass in the vicinity of 50 to $70 \mathrm{GeV}$. CDMS-II results show a 2-signal events with $0.6 \pm 0.1$ events as background. It may be possible that these events did not come from any real signal. In spite of this, in case we do not see the events as just background, a low recoil of 12.3 to $15.5 \mathrm{keV}$ could possibly indicate a light WIMP detection whose mass could not be too far away than $100 \mathrm{GeV}$. A large direct detection cross section where LSP is light (and that also satisfies the WMAP data) in a generic model like mSUGRA can be possible in a parameter space with a large $\tan \beta$ and nonvanishing trilinear coupling $A_{0}$. The relic density constraint is satisfied via h-pole resonant annihilation mechanism. Regarding direct detection $\tilde{\chi}_{1}^{0}-p$ cross section which reaches the level of the CDMS-II events, both the contributions involving the lighter as well as the heavier Higgs bosons are important. In this large $\tan \beta(=50)$ based scenario with Bino-dominated $\tilde{\chi}_{1}^{0}$, where light Higgs pole annihilation brings down the relic density to an acceptable level, the heavier Higgs boson contribution indeed dominates. This is in spite of the fact that $m_{H}$ can be larger by a factor of 3 to 4 compared to $m_{h}$.

Unlike the past analyses referred in this paper regarding the light Higgs-pole dominated LSP pair-annihilation processes for the cases with large values of $\tan \beta$, here we have worked on the direct detection prospects of the same region in relation to the recent interest of 
CDMS-II results. We have further shown in adequate detail the preference of only one sign of $A_{0}$ that gives feasibility to obtain the desired zone of light-Higgs pole annihilation for dark matter. We particularly perform an open analysis by considering non-MFV assumptions in relation to the $b \rightarrow s \gamma$ and $B_{s} \rightarrow \mu^{+} \mu^{-}$constraints, unlike most other recent works. We have considered the possibility of general flavor violation on $B_{s} \rightarrow \mu^{+} \mu^{-}$, specifically keeping in mind about the recent analyses as referred in this work. We believe that unless the MFVbased limits from flavor physics are violated by a very large extent, it is important not to discard appropriate scenarios that keep the region with the light Higgs-pole dominated pair-annihilation active. This is particularly relevant because of the ongoing and upcoming precision direct detection dark matter experiments and the advantage of having an associated light Higgs spectra as well as a relatively light overall spectra. In the last half of the paper we present a qualitative discussions on the interplay between collider searches for MSSM Higgs bosons $(h, H, A)$, as well as some other sparticles at the LHC and direct detection of dark matter for the aforementioned parameter space. In particular, we discuss the possibility of determining both $m_{\tilde{\chi}_{1}^{0}}$ and $m_{\tilde{\chi}_{2}^{0}}$ by combining the data from the direct detection of dark matter experiment with that of the edge in the $m_{\ell^{+} \ell^{-}}$distribution and the light Higgs boson mass measurement at the LHC.

We further note that because of $\tan \beta$ being large, copious production of $(H, A)$ in association with the $b$-quark would be possible at the LHC. Such an $H / A$ mass can be reconstructed by looking at the $H / A \rightarrow \tau^{+} \tau^{-}$decay mode. We also point out that large $\tan \beta$ and relatively low $m_{1 / 2}$ values of our benchmark points lead to a large number of multi-b jets in association with missing energy signal.

\section{Acknowledgments}

DD thanks P2I, CNRS for the support received as a post-doctoral fellow. DKG and MM acknowledge support from the Department of Science and Technology, India under grants SR/S2/HEP-12/2006 and SR/MF/PS-03/2009-VB-I respectively. DKG also thanks Theory Division, CERN for the hospitality when part of this work was done. 


\section{References}

[1] For reviews on Supersymmetry, see, eg, H. P. Nilles, Phys. Rep. 110, 1 ( 1984); H. E. Haber and G. Kane, Phys. Rep. 117, 75 ( 1985) ; J. Wess and J. Bagger, Supersymmetry and Supergravity, 2nd ed., (Princeton, 1991); M. Drees, P. Roy and R. M. Godbole, Theory and Phenomenology of Sparticles, (World Scientific, Singapore, 2005).

[2] T. P. Cheng and L. F. Li, Gauge Theory Of Elementary Particle Physics (Clarendon, Oxford, 1984, p.536).

[3] A. H. Chamseddine, R. Arnowitt and P. Nath, Phys. Rev. Lett. 49, 970 (1982); R. Barbieri, S. Ferrara and C. A. Savoy, Phys. Lett. B 119, 343 (1982); L. J. Hall, J. Lykken and S. Weinberg, Phys. Rev. D 27, 2359 (1983); P. Nath, R. Arnowitt and A. H. Chamseddine, Nucl. Phys. B 227, 121 (1983); N. Ohta, Prog. Theor. Phys. 70, 542 (1983); For reviews see [1] and P. Nath, R. Arnowitt and A.H. Chamseddine, Applied $N=1$ Supergravity (World Scientific, Singapore, 1984).

[4] D. J. H. Chung, L. L. Everett, G. L. Kane, S. F. King, J. D. Lykken and L. T. Wang, Phys. Rept. 407, 1 (2005); S. P. Martin, arXiv:hep-ph/9709356.

[5] G. Bertone, D. Hooper and J. Silk, Phys. Rept. 405, 279 (2005); G. Bertone and D. Merritt, Mod. Phys. Lett. A 20, 1021 (2005); J. Carr, G. Lamanna and J. Lavalle, Rept. Prog. Phys. 69, 2475 (2006).

[6] G. Jungman, M. Kamionkowski and K.Greist, Phys. Rep. 267, 195 (1996).

[7] J. R. Ellis, T. Falk and K. A. Olive, Phys. Lett. B 444, 367 (1998); J. R. Ellis, T. Falk, K. A. Olive and M. Srednicki, Astropart. Phys. 13, 181 (2000) [Erratum-ibid. 15, 413 (2001)]; A. Lahanas, D. V. Nanopoulos and V. Spanos, Phys. Rev. D 62, 023515 (2000); R. Arnowitt, B. Dutta and Y. Santoso, Nucl. Phys. B 606, 59(2001); T. Nihei, L. Roszkowski and R. Ruiz de Austri, JHEP 0207, 024 (2002); V. A. Bednyakov, H. V. Klapdor-Kleingrothaus and V. Gronewold, Phys. Rev. D 66, 115005 (2002).

[8] J. Edsjo and P. Gondolo, Phys. Rev. D 56, 1879 (1997).

[9] S. Mizuta and M. Yamaguchi, Phys. Lett. B 298, 120 (1993). 
[10] J. L. Feng, K. T. Matchev and T. Moroi, Phys. Rev. D 61, 075005 (2000); Phys. Rev. Lett. 84, 2322 (2000); J. L. Feng, K. T. Matchev and F. Wilczek, Phys. Lett. B 482, 388 (2000); J. L. Feng and F. Wilczek, Phys. Lett. B 631, 170 (2005); U. Chattopadhyay, T. Ibrahim and D. P. Roy, Phys. Rev. D 64, 013004 (2001); U. Chattopadhyay, A. Datta, A. Datta, A. Datta and D. P. Roy, Phys. Lett. B 493, 127 (2000); S. P. Das, A. Datta, M. Guchait, M. Maity and S. Mukherjee, Eur. Phys. J. C 54, 645 (2008), [arXiv:0708.2048 [hep-ph]].

[11] K. L. Chan, U. Chattopadhyay and P. Nath, Phys. Rev. D 58, 096004 (1998); U. Chattopadhyay, A. Corsetti and P. Nath, Phys. Rev. D 68, 035005 (2003).

[12] M. Drees and M. Nojiri, Phys. Rev. D47, 376 (1993); R. Arnowitt and P. Nath, Phys. Rev. Lett. 70, 3696 (1993); H. Baer and M. Brhlik, Phys. Rev. D53, 597 (1996), Phys. Rev. D 57, 567 (1998); H. Baer, M. Brhlik, M. Diaz, J. Ferrandis, P. Mercadante, P. Quintana and X. Tata, Phys. Rev. D63, 015007 (2000); J. R. Ellis, T. Falk, G. Ganis, K. A. Olive and M. Srednicki, Phys. Lett. B 510, 236 (2001); A. B. Lahanas and V. C. Spanos, Eur. Phys. J. C23, 185 (2002).

[13] E. Aprile et al. [XENON100 Collaboration], Phys. Rev. Lett. 105, 131302 (2010).

[14] Z. Ahmed et al. [The CDMS-II Collaboration], Science 327, 1619 (2010).

[15] I. Gogoladze, R. Khalid, S. Raza and Q. Shafi, arXiv:0912.5411 [hep-ph]; M. Asano, S. Matsumoto, M. Senami and H. Sugiyama, arXiv:0912.5361 [hep-ph]; M. Farina, D. Pappadopulo and A. Strumia, Phys. Lett. B 688, 329 (2010); X. G. He, T. Li, X. Q. Li, J. Tandean and H. C. Tsai, Phys. Lett. B 688, 332 (2010); M. Ibe and T. T. Yanagida, Phys. Rev. D 81, 035017 (2010); M. Kadastik, K. Kannike, A. Racioppi and M. Raidal, arXiv:0912.3797 [hep-ph].

[16] A. Bottino, F. Donato, N. Fornengo and S. Scopel, Phys. Rev. D 81, 107302 (2010); M. Holmes and B. D. Nelson, Phys. Rev. D 81, 055002 (2010); K. Cheung and T. C. Yuan, Phys. Lett. B 685, 182 (2010); J. Hisano, K. Nakayama and M. Yamanaka, Phys. Lett. B 684, 246 (2010); D. P. Roy, Phys. Rev. D 81, 057701 (2010); R. Allahverdi, B. Dutta and Y. Santoso, Phys. Lett. B 687, 225 (2010); D. Feldman, Z. Liu and P. Nath, Phys. Rev. D 81, 095009 (2010); J. Cao, K. i. Hikasa, W. Wang, 
J. M. Yang and L. X. Yu, JHEP 1007, 044 (2010); D. Feldman, Z. Liu, P. Nath and G. Peim, Phys. Rev. D 81, 095017 (2010); D. Feldman, Z. Liu and P. Nath, Phys. Rev. D 81, 117701 (2010).

[17] E. Komatsu et al. [WMAP Collaboration], Astrophys. J. Suppl. 180, 330 (2009), arXiv:0803.0547 [astro-ph].

[18] A. Djouadi, M. Drees and J. L. Kneur, Phys. Lett. B 624, 60 (2005).

[19] R. Barate et al. [LEP Working Group for Higgs boson searches], Phys. Lett. B 565, $61(2003)$.

[20] ATLAS collaboration, Detector and Physics performance technical design report, CERN-LHCC-99-15(1999), see http://atlas.web.cern.ch/Atlas/GROUPS/PHYSICS/TDR/TDR.html.

[21] I. Hinchliffe, F. E. Paige, M. D. Shapiro, J. Soderqvist and W. Yao, Phys. Rev. D 55, 5520 (1997) [arXiv:hep-ph/9610544]; V. D. Barger and C. Kao, Phys. Rev. D 60, 115015 (1999) [arXiv:hep-ph/9811489].

[22] J. R. Ellis, A. Ferstl and K. A. Olive, Phys. Lett. B 481, 304 (2000).

[23] M. W. Goodman and E. Witten, Phys. Rev. D 31, 3059 (1985); K. Greist, Phys. Rev. D 38, 2357 (1988); J. Ellis and R. Flores, Nucl. Phys. B 307, 883 (1988); R. Barbieri, M. Frigeni and G. Giudice, Nucl. Phys B 313, 725 (1989); A. Bottino et. al., Phys. Lett. B 295, 330 (1992); M. Drees and M. M. Nojiri, Phys. Rev. D 48, 3483 (1993); M. Drees and M. Nojiri, Phys. Rev. D 47, 4226 (1993) and Phys. Rev. D 48, 3483 (1993); M. A. Shifman, A. I. Vainshtein and V. I. Zakharov, Phys. Lett. B 78, 443 (1978); A. I. Vainshtein, V. I. Zakharov and M. A. Shifman, Usp. Fiz. Nauk 131, 537 (1980).

[24] P. Gondolo, J. Edsjo, P. Ullio, L. Bergstrom, M. Schelke and E. A. Baltz, JCAP 0407, 008 (2004).

[25] V. Sanglard et al. [The EDELWEISS Collaboration] Phys. Rev. D 71, 122002 (2005) [arXiv:astro-ph/0503265]. 
[26] V. A. Bednyakov and H. V. Klapdor-Kleingrothaus, Phys. Part. Nucl. 40, 583 (2009) [arXiv:0806.3917 [hep-ph]].

[27] M. Drees and M. Nojiri, Phys. Rev. D 48, 3483 (1993).

[28] H.E. Haber and Y. Nir, Phys. Lett. B306 327 (1993); H.E. Haber, hep-ph/9505240; A. Dobado, M.J. Herrero and S. Penaranda, Eur. Phys. J. C17 487 (2000) ; J.F. Gunion and H.E. Haber, Phys. Rev. D67 075019 (2003).

[29] Hisano et. al. of Ref. [16].

[30] G. Belanger, F. Boudjema, A. Cottrant, A. Pukhov and A. Semenov, Nucl. Phys. B 706, 411 (2005).

[31] For the latest limits on the sparticle masses from LEP experiments: see http://lepsusy.web.cern.ch/lepsusy/

[32] J. M. Frere, D. R. T. Jones and S. Raby, Nucl. Phys. B 222, 11 (1983); J. A. Casas, A. Lleyda and C. Munoz, Nucl. Phys. B 471, 3 (1996).

[33] M. S. Carena and H. E. Haber, Prog. Part. Nucl. Phys. 50, 63 (2003).

[34] U. Chattopadhyay, D. Das, A. Datta and S. Poddar, Phys. Rev. D 76, 055008 (2007).

[35] G. Degrassi, S. Heinemeyer, W. Hollik, P. Slavich and G. Weiglein, Eur. Phys. J. C 28, 133 (2003); B. C. Allanach, A. Djouadi, J. L. Kneur, W. Porod and P. Slavich, JHEP 0409, 044 (2004); S. Heinemeyer, hep-ph/0408340; Int. J. Mod. Phys. A 21, 2659 (2006); S. Heinemeyer, W. Hollik and G. Weiglein, Phs. Rep. 425,265,2006.

[36] A. L. Kagan and M. Neubert, Eur. Phys. J. C 7, 5 (1999); K. G. Chetyrkin, M. Misiak and M. Munz, Phys. Lett. B 400, 206 (1997) [Erratum-ibid. B 425, 414 (1998)]; P. Gambino and M. Misiak, Nucl. Phys. B 611, 338 (2001).

[37] M. Ciuchini, G. Degrassi, P. Gambino and G. F. Giudice, Nucl. Phys. B 527, 21 (1998); M. Ciuchini, G. Degrassi, P. Gambino and G. F. Giudice, Nucl. Phys. B 534, 3 (1998); G. Degrassi, P. Gambino and G. F. Giudice, JHEP 0012, 009 (2000). 
[38] S. Chen et al. [CLEO Collaboration], Phys. Rev. Lett. 87, 251807 (2001); B. Aubert et al. [BaBar Collaboration], [arXiv:hep-ex/0207076]; P. Koppenburg et al. [Belle Collaboration], Phys. Rev. Lett. 93, 061803 (2004).

[39] S. R. Choudhury and N. Gaur, Phys. Lett. B 451, 86 (1999); K. S. Babu and C. Kolda, Phys. Rev. Lett. 84, 228 (2000); A. Dedes, H. K. Dreiner, U. Nierste, and P. Richardson, Phys. Rev. Lett. 87, 251804 (2001); R. Arnowitt, B. Dutta, T. Kamon and M. Tanaka, Phys. Lett. B 538 (2002) 121; J. K. Mizukoshi, X. Tata and Y. Wang, Phys. Rev. D 66, 115003 (2002); S. Baek, P. Ko, and W. Y. Song, JHEP 0303, 054 (2003); T. Ibrahim and P. Nath, Phys. Rev. D 67, 016005 (2003); J.R. Ellis, K.A. Olive and V.C. Spanos, Phys. Lett. B 624, 47 (2005);

[40] T. Aaltonen et al. [CDF Collaboration], Phys. Rev. Lett. 100, 101802 (2008).

[41] A. Djouadi, M. Drees and J. L. Kneur, JHEP 0603, 033 (2006).

[42] For a recent article on MFV see for example: G. Colangelo, E. Nikolidakis and C. Smith, Eur. Phys. J. C 59, 75 (2009).

[43] K. i. Okumura and L. Roszkowski, Phys. Rev. Lett. 92, 161801 (2004).

[44] J. Foster, K. i. Okumura and L. Roszkowski, JHEP 0603, 044 (2006).

[45] T. Blazek and P. Matak, Nucl. Phys. Proc. Suppl. 198, 216 (2010).

[46] U. Chattopadhyay, D. Das, D.K. Ghosh and M. Maity, work in progress.

[47] The CMS Collaboration, J. Phys. G: Nucl. Part. Phys. 34, 995 (2007).

[48] D. A. Dicus and S. Willenbrock, Phys. Rev. D 39, 751 (1989).

[49] D. Dicus, T. Stelzer, Z. Sullivan and S. Willenbrock, Phys. Rev. D 59, 094016 (1999).

[50] C. Balazs, H. J. He and C. P. Yuan, Phys. Rev. D 60, 114001 (1999).

[51] F. Maltoni, Z. Sullivan and S. Willenbrock, Phys. Rev. D 67, 093005 (2003).

[52] R. V. Harlander and W. B. Kilgore, Phys. Rev. D 68, 013001 (2003).

[53] D. Choudhury, A. Datta and S. Raychaudhuri, arXiv:hep-ph/9809552. 
[54] C. S. Huang and S. H. Zhu, Phys. Rev. D 60, 075012 (1999).

[55] J. Campbell, R. K. Ellis, F. Maltoni and S. Willenbrock, Phys. Rev. D 67, 095002 (2003).

[56] J. J. Cao, G. P. Gao, R. J. Oakes and J. M. Yang, Phys. Rev. D 68, 075012 (2003).

[57] S. Dawson and C. B. Jackson, Phys. Rev. D 77, 015019 (2008).

[58] S. Dawson, D. Dicus and C. Kao, Phys. Lett. B 545, 132 (2002).

[59] B. Plumper, DESY-THESIS-2002-005.

[60] S. Dittmaier, M. 1. Kramer and M. Spira, Phys. Rev. D 70, 074010 (2004).

[61] S. Dawson, C. B. Jackson, L. Reina and D. Wackeroth, Phys. Rev. D 69, 074027 (2004).

[62] Z. Kunszt and F. Zwirner, Nucl. Phys. B 385, 3 (1992).

[63] E. Richter-Was, D. Froidevaux, F. Gianotti, L. Poggioli, D. Cavalli and S. Resconi, Int. J. Mod. Phys. A 13, 1371 (1998).

[64] C. Kao, D. A. Dicus, R. Malhotra and Y. Wang, Phys. Rev. D 77, 095002 (2008).

[65] C. Kao, S. Sachithanandam, J.Sayre and Y. Wang, Phys. Lett. B 682, 291 (2009). 